included optimism and self-efficacy towards a hoped-for self, but not of a feared self. The model explained $25 \%$ of the variance in SPA. Future research should explore how selfefficacy associated with possible selves could be targeted as an avenue for shaping SPA. Differences between hoped-for and feared selves may have implications for health behavior interventions, particularly those involving goals and motivation. It is possible that efforts to increase SPA should focus on enhancing self-efficacy towards achieving hoped-for selves, rather than of preventing feared selves.

\section{SENIOR LIVING EMPLOYEES' PERCEPTIONS OF AGING AND EMPLOYEE ENGAGEMENT}

J. Smith, Mather LifeWays

People who have negative perceptions of aging associate aging with an inevitable decline in physical, socioemotional, and cognitive functioning, whereas people who have positive perceptions of aging view aging as a time of continued development and maintenance of one's health and well-being. Drawing upon the job demands-resources model, positive perceptions of aging may be a resource for senior living employees that contributes to greater employee engagement. A total of 198 senior living employees (79\% female; $72 \%$ White; age: $\mathrm{M}=49.95, \mathrm{SD}=14.67$ ) completed a survey that included measures of perceptions of aging, work engagement, burnout, workplace satisfaction, purpose, and demographic information. Controlling for age, gender, education, and amount of interaction with residents, multiple regression analyses revealed that more positive perceptions of aging were associated with greater work engagement, lower burnout, and higher sense of purpose; however, perceptions of aging were unrelated to workplace satisfaction. In addition, mediation analyses examined whether the relationship between perceptions of aging and work engagement was mediated by sense of purpose. Using bias-corrected bootstrap estimation, the indirect effect of perceptions of aging on work engagement via purpose was statistically significant. These findings suggest that positive perceptions of aging may be a resource for senior living employees, and these positive attitudes toward aging may contribute to greater work engagement by cultivating a greater sense of purpose.

\section{THE AGING SEMANTIC DIFFERENTIAL PREDICTS CONTACT WITH OLDER ADULTS AND KNOWLEDGE OF AGING}

\section{R. Intrieri, Western Illinois University}

Previous studies have demonstrated a significant relationship between attitudes toward aging and knowledge of aging and contact with older adults. However, most of these studies are limited by their experimental design. Previous studies examined how attitudes may influence knowledge and contact with older adults without considering verbal ability as a potential covariate. Vocabulary is generally thought to be the best single indicator of intelligence and is included in most assessments of intellectual functioning. The current study assesses the relationships between the four factors of Aging Semantic Differential (ASD; Instrumentality, Autonomy, Acceptability, and Integrity) and knowledge of aging and frequency of contact with older adults while using biological sex, age, and verbal ability as a covariates. The convenience sample consisted of 471 undergraduate students, with a mean age of $19.68(\mathrm{SD}=2.278)$. Two hierarchical regression models were used to examine how well the ASD predicted knowledge of aging and self-reported contact with older adults while controlling for verbal ability. A hierarchical regression model using knowledge of aging as the dependent variable was statistically significant $F(5,465)=10.386$, $\mathrm{p}<0.0001, \mathrm{R} 2=.10$ with Verbal Ability $\beta=0.219, \mathrm{p}<0.0001$ and ASD-Integrity $\beta=0.219, \mathrm{p}<0.0001$ as significant predictors. Regression model two used contact with older adults as the dependent variable was statistically significant $\mathrm{F}(6$, 462) $=12.523, p=.023, R 2=.031$. Significant effects were only observed for ASD-Instrumental, Acceptance, and Integrity ( $\beta=-0.115, p=0.041$ Acceptance $\beta=-0.155, p=0.019$; Integrity $\beta=0.160, p=0.022$, respectively). This study provides evidence that verbal ability influences knowledge of aging but does not predict contact with older adults.

\section{WHAT DOES AGE FRIENDLY MEAN? AN EXPLORATION THROUGH INTERGENERATIONAL ART}

D. Bradley, Western Kentucky University

Using generativity theory to explore the meaning of "agefriendly" in a community setting, this paper explores how multiple generations planned and celebrated a community's livability and age-friendliness. Bowling Green, KY, a vibrant university community, and one of the original seven US cities to join the World Health Organization (WHO) Age-Friendly Cities and Communities Initiative, has demonstrated that a cohesive community is friendly to all ages. This project brought together citizens across the lifespan to create a visual mural celebrating the accomplishments of Bowling Green. Four cohorts $(\mathrm{n}=122)$ of the Bowling Green, KY Age-Friendly Cities Over 50 Academy participated in focus groups to develop themes around the prompt "What does living in an age-friendly community mean to you?" Major themes identified included "not being invisible", "connecting with others" and "continuing to contribute." Concept sketches were developed by the mural artist and shared during a community meeting of key stakeholders in the city, a meetings of college students through the university sponsored Companions of Respected Elders volunteer group and a reunion gathering of older adults involved in the age-friendly cities project. After reflective dialog, a mural design was selected and completed over two days using hands-on-efforts of multiple individuals from these groups. This presentation concludes with a discussion of best practices towards replication in other communities.

\section{AGING PERCEPTIONS: INVESTIGATION OF DIFFERENCES AT THREE DECADES}

N. Meinertz, J. Margrett, M. Shelley, Iowa State University

Ageism, the discrimination or prejudice towards an individual based on age (Butler, 1969), is understood to be both an individual and a societal issue. Ageism and related negative age perceptions can have an impact on personal health (Levy, Zonderman, Slade, \& Ferrucci, 2009) and are influenced by societal shifts in ideologies as well as individual experiences (Rippon, Zaninotto, \& Steptoe, 2015). Using bioecological (Gans \& Silverstein, 2006) and life course (White, Klein, \& Martin, 2015) theories, we examined group differences in chronological perceptions of "old" 
for three distinct measurement timepoints $(1974,1981, \&$ 1999/2000). The sample included 6,765 U.S. respondents, with 1,554 respondents from 1974, 2,425 respondents from 1981, and 2,786 respondents from 1999/2000. Analysis of variance models were estimated to determine whether respondent demographics had significant impacts on the chronological perceptions of "old" for target males and females. Statistically significant effects were found for age $(\mathrm{F}[2,6,746]=112.77, \mathrm{p} \leq 0.001), \operatorname{sex}(\mathrm{F}[2,6,746]=84.59$, $\mathrm{p} \leq 0.001)$, education $(\mathrm{F}[8,13,492]=10.53, \mathrm{p} \leq 0.001)$, race $(\mathrm{F}[2,6,746]=29.17, \mathrm{p} \leq 0.001)$, and measurement timepoints $(\mathrm{F}[4,13,492]=82.81, \mathrm{p} \leq 0.001)$, as well as several interactions indicating differences within response categories of the independent variables. Results indicate that perceptions are more complex than demographic variables. In addition to historical and societal shifts, future research should consider other aspects of perceptions, such as aging anxiety and past experiences.

\section{ALL THE SINGLE LADIES': PERSPECTIVES ON IDENTITY AND WELL-BEING ACROSS THE LIFESPAN}

A. Fisher ${ }^{1}$, D. Cloutier ${ }^{2}, 1$. Department of Psychology,

University of Victoria, 2. University of Victoria

Singlehood may or may not be a choice; but it is often a statement about identity for many women. Historically, singlehood has been a deviant identity. One that signals a personal flaw or eccentricity. However, high divorce rates, and increasing preferences for cohabitation and living alone have made singlehood increasingly common across societies in the Global North. Further, while a large body of research reports on the health benefits that accrue from non-single status, little research has focused on the heterogeneity among single individuals as a growing cohort, and on the ways that singlehood both shapes identity, and influences health and well-being. Moreover, only a limited body of research has explored how this phenomena is changing societally, and how it has affected women of different ages and life stages. Using a life course perspective and a critical feminist lens, this paper draws upon qualitative interviews to understand the 'lived experience of singlehood' for women at three different life stages notably as they seek educational opportunities and enter the job market, as middle-aged members of the workforce and mothers, and finally, as persons exiting the workforce or retirees. Our findings suggest that women in each of these stages experiences singlehood in unique ways. From our critical lens, economic well-being is consistently important for singles as is the nature, rather than the number of friendships. This research is a starting point for understanding the implications of growing numbers of single women within a dynamic and historic period in time.

\section{CHALLENGING AGEIST ATTITUDES FOR ALL INTENTS AND PURPOSES: A MIXED METHODS ANALYSIS}

K. Carden ${ }^{1}$, S. Letang 2 , J. Choi², M. Hull2 , T. Popp², L. Whitley ${ }^{2}$, D. Potts ${ }^{2}$, R. Allen ${ }^{2}$, 1. University of Alabama,

2. The University of Alabama

Service learning programs, such as Bringing Art to Life (BATL), are designed to address ageist attitudes by bolstering empathetic intergenerational relationships, creating a comprehensive, realistic view of aging, and fostering positive attitudes towards older adults. This project examined learning outcomes for students enrolled in an experiential University of Alabama Honors College course (BATL), wherein students work directly with persons with dementia (PWD) to produce a life story incorporating art. Students completed electronic surveys measuring empathy, mindfulness, and attitudes toward older adults generally, individuals with dementia, and interest in community service. A series of within-subjects analyses of covariance revealed increases in empathy and improved attitudes toward older adults and PWD. Students also kept journals documenting their experiences. In a sequential mixed methods design, a qualitative phase II follow-up explanations model (Creswell et. al., 2003) was employed to explain and expand on quantitative results. Student participant journals were collected, a codebook and coding system was developed, and four coders utilized an iterative hermeneutic coding process to enhance inter-rater reliability. Overall, the integrated mixed-methods results provide evidence for the mechanisms that facilitate the change in students' attitudes. Specifically, existential awareness fosters mindfulness and empathy that subsequently facilitates change in ageist attitudes generally. Moreover, meaningful engagement with the present moment with other group members versus meaningful engagement with art was a more powerful predictor of intergenerational relationship building, conversations regarding purpose- and meaning-inlife, and subsequent shifts in ageist attitudes.

\section{DO FEELINGS AND KNOWLEDGE ABOUT AGING PREDICT AGEISM?}

C. Cooney ${ }^{1}$, J. Minahan ${ }^{2}$, K. Siedlecki², 1. Fordham

University, Psychology Department, Bronx, NY, 2. Fordham

University, Psychology Department, Bronx, NY, USA

Ageism against older adults is a prevalent issue in the U.S. and has consistently been found to negatively impact older adults (e.g., Sargent-Cox, 2017). Research has shown that being exposed to ageism (in the form attitudes or behaviors) is related to diminished cognitive performance, psychological well-being, self-esteem, and worse self-perceptions of aging among older adults (e.g., Han \& Richardson, 2015; Robertson et al., 2016). Therefore, it is important to understand what factors predict ageism. In the current study, aging anxiety, knowledge of aging, death anxiety, and intergenerational contact frequency and quality were examined as predictors of ageism in a large sample of individuals $(\mathrm{N}=419)$ between the ages of 18 and 86 years via an online survey. The average age of participants was 46.09 ( $\mathrm{SD}=19.28), 79.50 \%$ were female, and $85.70 \%$ were White. Using hierarchical regression analyses, we found that higher levels of aging anxiety and lower levels of knowledge of aging, frequency of contact, and quality of contact were related to higher levels of ageism beyond the influence of demographic and well-being factors (e.g., positive and negative affect, depression, life satisfaction). Although death anxiety was significantly related to ageism, the relationship was fully mediated by aging anxiety. Finally, knowledge of aging, frequency of contact, and quality of contact were examined as moderators, and we found that knowledge of aging buffered the impact of aging 